$\begin{array}{ll}\text { le portiQue } & \text { Le Portique } \\ \text { Revue de philosophie et de sciences humaines }\end{array}$

$5 \mid 2000$

Passages du siècle

\title{
Il est midi docteur Mies
}

Description d'un espace rayonnant : le pavillon de Barcelone de Mies van der Rohe

Jean-Pierre Marchand

\section{(2) OpenEdition}

12 Journals

Édition électronique

URL : http://journals.openedition.org/leportique/400

DOI : $10.4000 /$ leportique.400

ISSN : $1777-5280$

Éditeur

Association "Les Amis du Portique"

Édition imprimée

Date de publication : 1 janvier 2000

ISSN : 1283-8594

Référence électronique

Jean-Pierre Marchand, « II est midi docteur Mies », Le Portique [En ligne], 5 | 2000, mis en ligne le 24

mars 2005, consulté le 26 mars 2021. URL : http://journals.openedition.org/leportique/400 ; DOI :

https://doi.org/10.4000/leportique.400

Ce document a été généré automatiquement le 26 mars 2021.

Tous droits réservés 


\section{Il est midi docteur Mies}

Description d'un espace rayonnant : le pavillon de Barcelone de Mies van der Rohe

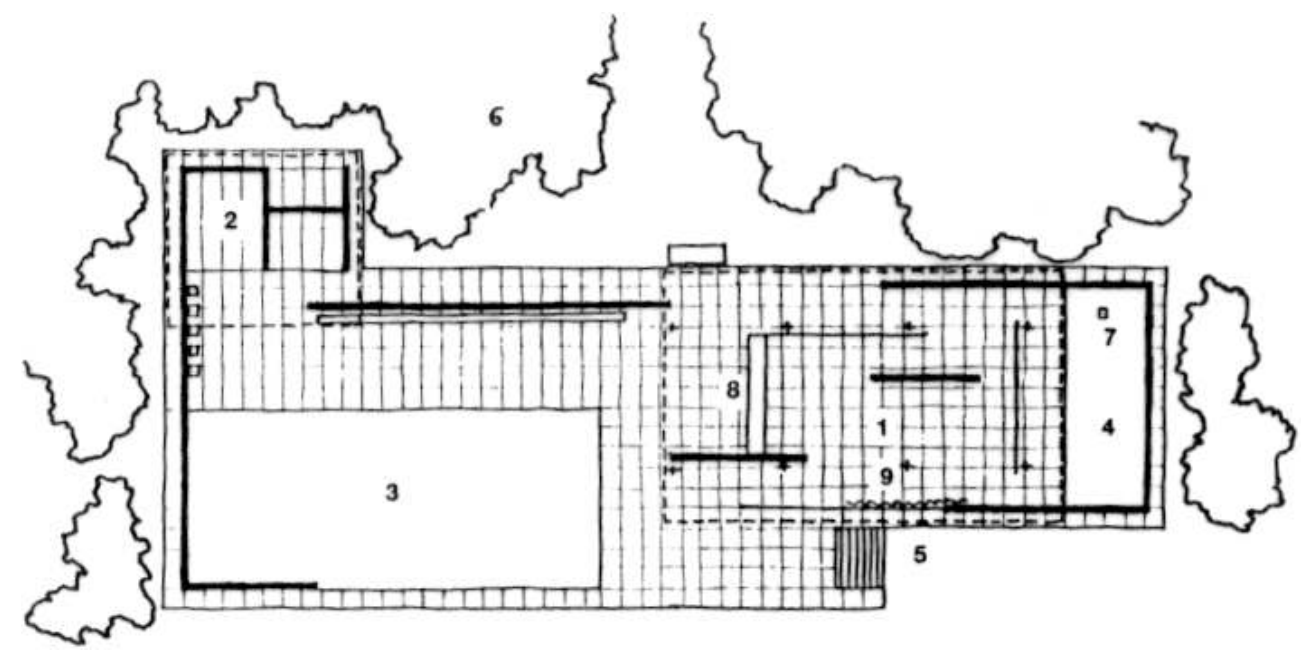

Fig. 1
1. Pavillon principal
2. Petit pavillon (billetterie)
3. Grand bassin
4. Petit bassin
5. Entrée principale
6. Jardin
7. Statue de Kolbe
8. Boîte de lumière
9. Rideau rouge

Ce n'est pas seulement comme chef-d'œuvre emblématique que le pavillon de Barcelone de Mies van der Rohe mérite une place à l'ombre de ce Portique consacré au xxe siècle. Dans un article dont la traduction française vient de paraître dans Le Visiteur, Robin Evans ${ }^{1}$ souligne l'ambivalence de la réception de ce symbole d'une 
«Allemagne nouvelle » qu'a été le pavillon au cours de l'exposition universelle de 1932. Certains ont vu en lui un manifeste de cette modernité que le nazisme liquidera au prétexte de dégénérescence; d'autres le signe avant-coureur de la victoire des hordes hitlériennes.

2 Responsable du Bauhaus dans les premières années du pouvoir hitlérien, Mies a effectivement espéré qu'une telle promesse de "renaissance» opterait pour une modernité esthétique fondée sur la rationalité et les techniques nouvelles. Mais Hitler n'aimait pas le pavillon. Ayant dessiné lui-même certains des symboles les plus caractéristiques de son mouvement il lui a été impossible d'accepter que ses pouvoirs de metteur en scène - «Ein Volk, ein Reich, ein Führer " - composassent avec des créateurs d'avant-garde. Il se fit, avec la complicité de son alter ego l'architecte Albert Speer, le doctrinaire simpliste mais efficace d'un néoclassicisme mâtiné de folklore. Et c'est en Amérique, notamment à Chicago, que Mies, bien avant von Braun, trouva une culture de l'espace favorable à ses idées. Cette migration du créateur du pavillon de l'Allemagne anticipe, quand bien même il aura souhaité devenir un des architectes du nouveau Reich, la défaite future du régime. Peut-être s'est-il agi d'une sorte de quiproquo. Car l'événement se reconstruit comme un symptôme. Mies aurait rêvé d'une nouvelle civilisation, et notamment d'une Allemagne moderne guérie des graves traumatismes que l'industrie et la technique avaient infligé à son âme romantique ${ }^{2}$. Mais voilà ; le nazisme était beaucoup plus régressif, nihiliste et fuite-en-avantiste qu'il paraissait. Ses hordes campaient dans le refus vertigineux de la civilisation. L'exacerbation des passions racistes et en particulier de l'antisémitisme auraient dû servir d'alerte. On sait qu'un grand penseur comme Heidegger n'a pas vu - n'a pas voulu voir? - cela même qui montrait clairement comment la noblesse de ce qu'il appelle lui-même le mouvement ne pouvait que s'avilir et sombrer dans l'ignoble. C'était payer très cher, et d'abord pour les victimes, cette concession cynique et altière faite au principe de la «mobilisation des masses $»^{3}$.

3 Nous voilà bien! Mais la " volonté d'art » si chère à Aloïs Riegl, quand elle est à ce point une volonté de beauté, ne se déploie-t-elle pas souvent entre barbarie et civilisation? Dans sa tension entre la destruction d'un ordre établi contre lequel elle proteste et la construction de cet ordre nouveau dont elle forme le rêve, elle ne pouvait être que tentée par l'acceptation de la violence.

4 Je risquerais alors cette hypothèse. Staline aurait voulu le pacte germano-soviétique pour une double raison de fond: donner le temps au nazisme de détruire tous les germes d'un socialisme voire d'un communisme démocratique notamment en Europe centrale et laisser l'Allemagne devenir un «ennemi intéressant ». Je veux dire par là que la mystification stalinienne avait tout intérêt, en jouant sur la double fibre patriotique et révolutionnaire, à combattre héroïquement puis à triompher de l'ennemi mortel de la " patrie du socialisme ». En ce sens le nazisme fut un cadeau inespéré à la mystification stalinienne. On sait ce qu'il advint : transformation de l'Europe de l'Est en colonies et en glacis de l'Empire puis, surtout, construction du mythe d'un Parti héroïquement anti-nazi. Le rêve! pour des aventuriers totalitaires pour lesquels l'aspiration révolutionnaire n'était qu'un fond de commerce destiné à conférer de la dynamique - et de la chair à bureaucrates - aux stratèges d'appareil. Il y a une gémellité entre nazisme et stalinisme qui a surtout profité au second. Au fond les légions anticommunistes d'Adolf étaient une bêtise. La destruction du stalinisme passait d'autant moins par un corps à corps guerrier qu'il s'était débrouillé pour s'en 
nourrir en y trouvant notamment de quoi dissimuler pour quelques décennies son échec et son imposture. Je ne ferai pas ici la liste de tous les avantages que cette naïveté a servi sur un plat en or à la mystification stalinienne.

5 Pour aller vite, c'est la conquête de la lune, plus généralement la guerre des étoiles qui finirent pas faire craquer la combinaison de menteur dans laquelle s'était glissé le système stalinien. Le ridicule tragique de Tchernobyl fut un moment décisif dans cette guerre froide livrée à coups de grands spectacles mondiaux high tech. Je veux dire surtout que c'est Mies qui, avec son pavillon, avait raison contre Hitler et ses légions. Mies est le créateur, à Chicago, d'un très célèbre gratte-ciel. Je soutiens que ce n'est pas faire un rapprochement simpliste que de le comparer aux fusées créées par von Braun. Celles-ci, surtout, ont été beaucoup plus efficaces contre l'Union Soviétique que toute la Wehrmacht sous contrôle SS. L'alliance anti-nazie, puis la possession par l'URSS ellemême de l'arme nucléaire ont empêché l'utilisation de celle-ci contre le système stalinien. C'est le déclenchement de la guerre froide et, corrélativement, la mise en place d'un terrain de guerre symbolique qui purent dénouer l'impasse dans laquelle s'était fourvoyé l'ensemble de "l'occident ». À mes yeux le pavillon de Mies ne cesse de proclamer sa victoire contre les crimes mobilisateurs, mais contre-productifs, du nazisme.

6 Formons alors symboliquement la « croix » du siècle : le pavillon de Mies - transfuge d'avant-guerre - pour la branche horizontale; une fusée de Von Braun - transfuge d'après-guerre - pour la branche verticale. Faut-il se lamenter sur le fait que le vampire stalinien a plongé le socialisme révolutionnaire non seulement dans l'abjection mais aussi dans la bêtise?

D'un éblouissement l'autre

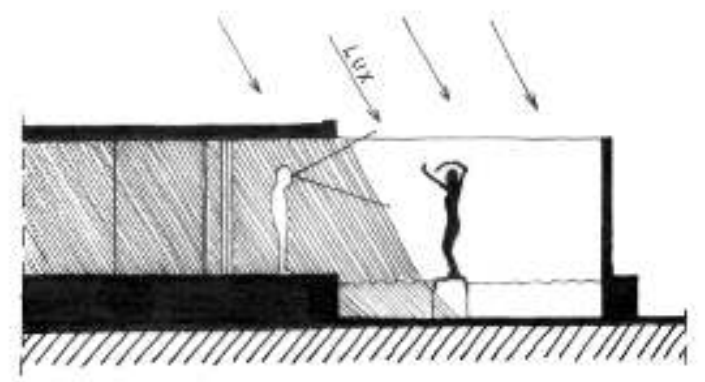

Fig. 2

7 Contre toute attente, je vais commencer la visite par la statue de Georg Kolbe intitulée Le Matin. L'usage veut que cette œuvre, qui est installée dans le plus petit bassin du pavillon, soit considérée comme un exemple négligeable de réalisme aryenisant. Il est vrai que ce n'est pas elle que l'on voit tout d'abord mais l'écrin dans laquelle elle se niche et qui n'est autre que l'œuvre même de Mies. Le contraste est cependant révélateur entre l'académisme de l'une et le purisme avant-gardiste de l'autre. Je tiens cependant que la statue de Kolbe n'est pas insignifiante. Elle exprime dans un langage allégorique ce qu'il en est de l'architecture selon Mies : une poétique de la lumière.

La statue ne tend-elle pas en effet les mains en direction du soleil pour se protéger d'une trop vive lumière? Il y aura toujours, tant le contraste est fort entre l'ombre 
projetée par la dalle du toit et la large fente de lumière qui ouvre sur le ciel au-dessus de la statue quelque motif pour être ébloui.

L'éblouissement se produit quand le regard, au lieu de s'habituer progressivement à une nouvelle intensité lumineuse, passe rapidement de l'ombre à la clarté. Une trop grande quantité de lumière peut nous aveugler. Cependant, quand bien même la lumière serait-elle relativement faible il suffit, pour nous éblouir, qu'elle surprenne un regard habitué à l'ombre.

10 On se représente la lumière comme la condition essentielle de la visibilité des choses. Elle est ce par quoi notre champ visuel reconnaît et détaille le visage du monde. L'aveugle, qui vit l'absence de sensibilité visuelle à la lumière dans un monde lumineux et visible sait ce qu'il en coûte de ne pas voir. L'éblouissement est en revanche une manière de voir et de ressentir la lumière comme telle. En m'empêchant momentanément de voir les choses que la lumière pourtant éclaire il me fait percevoir cela même qui les rend visibles. Des automobilistes sont morts pour avoir vu, à en être éblouis, la lumière des phares au lieu de la route qu'ils ont pour mission d'éclairer.

11 Mais l'éblouissement dit encore autre chose : la douleur plus ou moins intense qu'il provoque - nous pouvons ainsi avoir vraiment mal aux yeux - nous révèle notre "photo-sensibilité ». Nous sommes affectés par un brusque changement de lumière. Mais l'éblouissement ne consiste pas en un simple afflux massif de lumière dans l'intériorité vivante du sujet. La lumière qui m'éblouit n'entre pas dans ma conscience comme un rayon à travers la fenêtre d'une chambre d'abord mal éclairée. L'éblouissement pince les yeux en tant qu'organes. Ils ne sont plus des trous, ou des fenêtres, mais des être vivants qui me signifient que mon corps tout entier est toujours déjà du côté du monde et de sa chair.

Le monde est lumineux, nous dit la statue, il est un monde-lumière. Nous ne sommes pas à la fenêtre de notre conscience pour voir à travers des rideaux un spectacle de monde incidemment éclairé par la lumière. Par nos yeux nous sommes nous-mêmes une partie de ce monde lumineux. L'éblouissement nous amène bien au-delà de la simple « réflexion » : il nous plonge brusquement dans un fiat lux originaire du monde. Notre être monadique n'a pas de fenêtre, n'a pas de trou pour voir. Les yeux ne sont pas un « regard », mais des globes captifs de la luminosité du monde.

13 La sculpture, qui se tient d'abord cachée et à l'abri de nos regards derrière la paroi de marbre vert du bloc à droite du pavillon, est une allégorie de l'essence lumineuse du monde et de l'art comme connaissance emphatique de ce monde-lumière. L'éblouissement, qui est l'expérience d'un excès brusque et relatif de lumière, d'une chute soudaine mais révélatrice dans la luminosité du monde, me signifie ma propre essence lumineuse. Mes yeux ne sont pas des vides mais des boules de lumière protégées par un corps, médités par une pensée, et qui sont comme l'archéologie des premiers âges de la vie. La lumière, qui a permis la vie, n'a jamais abandonné le vivant. Elle le tient au monde par les yeux, où brille l'antique liquide de la mer primordiale.

La statue éblouie ne voit rien, ne voit plus ou pas encore, mais éprouve non sans douleur la luminosité essentielle du monde.

Cette douleur est celle-là même de la connaissance.

16 L'art de Mies nous montre le monde dans son midi, ce moment où le matin tient la main au plus clair et au plus chaud de la journée. 
pas un objet «concret „éclairé par la lumière, mais un ensemble jouant une ceuvre de lumière, dans un monde de lumière et pour des yeux d'abord éblouis. Nourri d'une histoire pluri-millénaire, le pavillon ne se laisse pas enfermer dans une image-cliché d'architecture. C'est aussi par là qu'il apparaît abstrait au premier regard. Rien n'est 
plus concret, cependant, que le jeu de ses instruments avec la lumière. Ce jeu est esthétique, sensuel, musical. Le soleil n'est pas un projecteur mais, de manière poétiquement platonicienne, le symbole du bien. Que l'œuvre traduit en beauté.

L'entrée du pavillon : une séquence initiatrice

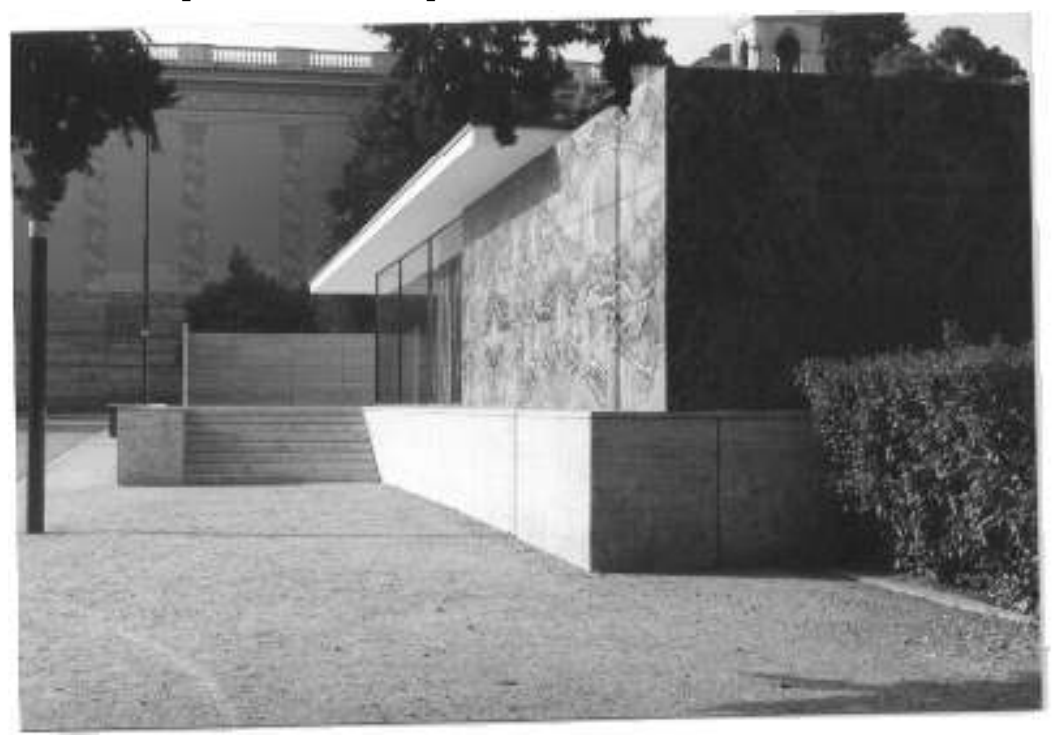

\section{NOTES}

1.. Le Visiteur $\mathrm{n}^{\circ} 4$, Société française des architectes, Paris, 1999.

2.. «Irruption de la machine dans un pays qui avait jusqu'à présent possédé une culture. Je voyais tomber la mort sur une vie d'une infinie beauté », notait Mies van der Rohe en 1927 en citant Guardini. In " Mies van der Rohe. Réflexions sur l'art de bâtir », Le Moniteur, Paris, 1996, p. 278.

3.. La Shoah constitue par elle-même un événement sinon l'Événement. Je suggère seulement que la volonté d'exterminer le peuple qui « est sorti d’Égypte » est conforme à la vision totalitaire de la « raison » nazie. Ne perdons pas de vue que, corrélativement, le stalinisme a instrumentalisé l'opposition à l'antisémitisme. Les juifs soviétiques n'étaient reconnus dans leur droit que de manière conditionnelle. 


\section{RÉSUMÉS}

À suivre certains historiens comme Fritz Neumeyer, le pavillon de Barcelone, construit en 1929 par l'architecte allemand Mies van der Rohe pour l'exposition universelle, est l'œuvre architecturale la plus extraordinaire du $\mathrm{xx}^{\mathrm{e}}$ siècle. S'il est voué à l'exposition, à la représentation, à la «diplomatie culturelle ", il est surtout habité par une méditation d'une rare intensité. Il est une méditation construite sur le thème de ce que doit être l'architecture à une époque qui a vu, comme l'écrivait Guardini cité par Mies lui-même dans ses carnets en 1927, « tomber la mort sur une vie d'une infinie beauté ".

Notre contribution est une visite méditative au cours de laquelle nous avons rencontré toute l'importance qu'avait la lumière pour Mies. Elle est bien ce qui « révèle les masses bâties à la vie». 